\title{
PERFIL EPIDEMIOLÓGICO DAS NOTIFICAÇÕES DE VIOLÊNCIA CONTRA O IDOSO NO PARANÁ
}

\author{
Fernanda Shizue Nishida $^{1}$; Mateus Dias Antunes ${ }^{2}$. \\ ${ }^{1}$ Enfermeira, doutora em Ciências pela Universidade de São Paulo, professora do \\ Programa de Mestrado em Promoção da Saúde do Centro Universitário de Maringá \\ - UNICESUMAR; Pesquisadora do Instituto Cesumar de Ciência, Tecnologia e \\ Inovação (ICETI) - Maringá, Paraná, Brasil. fernanda_nishida@hotmail.com \\ ${ }^{2}$ Fisioterapeuta, mestre em Promoção da Saúde do Centro Universitário de Maringá \\ - UNICESUMAR; Bolsista da Coordenação de Aperfeiçoamento de Pessoal de Nível \\ Superior (CAPES) - Maringá, Paraná, Brasil.
}

Recebido em: 02/10/2017 - Aprovado em: 21/11/2017 - Publicado em: 05/12/2017 DOI: 10.18677/EnciBio_2017B126

\begin{abstract}
RESUMO
A violência contra 0 idoso está presente em todas as classes sociais e tem relevantes consequências na saúde dessa população. $O$ objetivo do presente estudo foi traçar o perfil epidemiológico das notificações de violência contra o idoso no estado do Paraná. Estudo transversal, descritivo de abordagem quantitativa que utilizou dados do Sistema de Informação de Agravos de Notificação (SINAN) do Departamento de Informática do SUS (DATASUS), do Ministério da Saúde. A população de estudo foi constituída por todas as 793 notificações de violência contra idosos residentes no estado do Paraná. Verificou-se que as notificações foram mais frequentes nas mulheres brancas de baixa escolaridade, o local de ocorrência em que mais ocorre a violência foi o ambiente domiciliar tendo o filho(a) como principal agressor. A violência física foi o tipo mais comum através de força física/espancamento. O encaminhamento dos casos em sua maioria foi ambulatorial com evolução para alta, embora óbitos tenham ocorrido numa parcela menor. Conclui-se que a violência contra o idoso é uma questão de saúde pública, frequente e muitas vezes oculta na sociedade. Estudos desse tipo trazem a luz características importantes para compreensão do problema e podem auxiliar na elaboração e planejamento de ações e políticas públicas destinadas ao controle e redução desse problema social e de saúde publica.
\end{abstract}

PALAVRAS-CHAVE: Envelhecimento, Exposição à Violência, Epidemiologia, Promoção da Saúde.

\section{EPIDEMIOLOGICAL PROFILE OF NOTIFICATIONS OF VIOLENCE AGAINST THE ELDERLY IN PARANÁ}

\begin{abstract}
Violence against the elderly is present in all social classes and has relevant consequences for the health of this population. The objective of the present study was to outline the epidemiological profile of reports of violence against the elderly in the state of Paraná. A cross-sectional, descriptive study of a quantitative approach
\end{abstract}


that used data from the SINAN (Information System for Notifiable Diseases) of the Department of Information Technology of SUS (DATASUS) of the Ministry of Health. The study population consisted of all 793 reports of violence against elderly people living in the state of Paraná. It was verified that the notifications were more frequent in white women with low schooling, the place of occurrence in which the most violence occurred was the home environment with the child as the main aggressor. Physical violence was the most common type through physical force / beating. The majority of the cases were outpatients with progression to discharge, although deaths occurred in a smaller portion. It is concluded that violence against the elderly is a public health issue, frequent and often hidden in society. Studies of this kind bring important characteristics to understanding the problem and can help in the elaboration and planning of actions and public policies aimed at controlling and reducing this social and public health problem.

KEYWORDS: Aging, Violence Exposure, Epidemiology, Health Promotion.

\section{INTRODUÇÃO}

O envelhecimento populacional atualmente representa um fenômeno mundial, e no Brasil tem ocorrido de maneira acelerada (WARMLING et al., 2017), e iniciou em países desenvolvidos e vem crescendo exponencialmente nos países em desenvolvimento. Nesse cenário, a violência contra o idoso está presente em todas as classes sociais e tem relevantes consequências na saúde dessa população (APRATTO JÚNIOR, 2010), tornando-se um desafio para a saúde pública, à medida que impõe a necessidade de políticas sociais e novos direcionamento para atenção integral à saúde do idoso (DUQUE et al., 2012).

A violência de acordo com a literatura científica reduz a expectativa de vida para a vítima idosa (OLIVEIRA et al., 2013). Violência representa o uso da agressividade de forma excessiva e intencional para ameaçar ou cometer alguma ação que resulte em trauma psicológico, acidente ou morte (SAMPAIO et al., 2017). Ela abrange todos os atos de violação dos direitos civis, sociais, econômicos, culturais e políticos (CACHINA et al., 2016).

Ainda que existam diferenças metodológicas das pesquisas e da tipologia de classificação de violência utilizada nas mesmas, os achados evidenciam a relevância da situação de violência contra idosos no âmbito nacional e internacional (SIEGEL et al., 2017). Em estudo em 524 cidades brasileira evidenciou que 67,7\% dos idosos foram vítimas de violências físicas e $29,1 \%$ de psicológica (MASCARENHAS et al., 2012). Em Recife, pesquisa conduzida em uma unidade de saúde, constatou que entre os entrevistados $20,7 \%$ dos idosos relataram ter sofrido pelo menos um tipo de violência (DUQUE et al., 2012). No âmbito internacional, nos Estados Unidos, entre 5.777 idosos entrevistados, 11,4\% relataram ter sido vítimas de violência, dos quais $4,6 \%$ dessas foram psicológicas e 1,6\%, física (ACIERNO et al., 2010). Salienta-se que exista dificuldade em mensurar a prevalência de violência em virtude das definições e dos conceitos que apresentam alterações entre culturas e setores (IMSPM, 2007).

Diversos fatores estão associados a violência contra os idosos dentre eles sexo feminino, faixa etária de 60 a 69 anos, estado civil casado e morar acompanhado (PAIVA; TAVARES, 2015).

Além de conhecer estes fatores, serviços de atenção primária são estratégias para lidar com casos de violência contra o idoso, uma vez que funcionam com a porta entrada dos serviços públicos de saúde (WANDERBROOCKE; MORÉ, 2013). 
Nesse contexto, os profissionais de saúde têm um relevante papel no enfrentamento da violência, na prevenção, identificação precoce e/ou cuidado do idoso vitimado (WANDERBROOCKE; MORÉ, 2012). Cabe evidenciar que esses profissionais devem também promover a saúde dos idosos, empoderando-os para lidar com possíveis casos de violências (SOUSA et al., 2016).

São escassos, estudos atuais com os dados sobre a violência contra o idoso no Paraná, por conseguinte, este estudo tem intuito de auxiliar a comunidade acadêmica e os serviços de saúde do estado do Paraná a proporem espaços e práticas que promovam a saúde dos idosos que sofrem violência no contexto da interdisciplinaridade. Este estudo pode contribuir com os Conselhos Estaduais do Idoso das cidades do Paraná para que possam ter informações relativas ao perfil da violência contra o idoso visando então um direcionamento das ações. Nesse sentido, este estudo tem por objetivo traçar o perfil epidemiológico das notificações de violência contra o idoso no estado do Paraná.

\section{MATERIAL E MÉTODOS}

Foi utilizado um estudo descrito, retrospectivo com dados do Sistema de Informação de Agravos de Notificação (SINAN) do Departamento de Informática do SUS (DATASUS), do Ministério da Saúde. Foram obtidos dados de todas as notificações de violência contra o idoso (idade igual ou superior a 60 anos) no Paraná, ocorridas entre 01 de janeiro a 31 de dezembro de 2014. No total, foram 793 notificações realizadas neste período, nesta população.

As variáveis de estudo foram: raça, escolaridade, tipo de violência (violência física, psicológica ou moral, violência sexual, tortura, espancamento e enforcamento), agressão com objetos pérfuro-cortantes, contundentes, lesão por substancia ou objeto quente, envenenamento, lesão por arma de fogo, ameaça, assédio sexual e estupro; quem foi o autor da violência (pai, mãe, cônjuge, filho (a), irmão, amigo ou conhecido, cuidador ou a própria pessoa). Qual o tipo de encaminhamento foi dado (ambulatorial ou hospitalar) e evolução dos casos (alta, evasão/fuga).

Essas variáveis constam na ficha de notificação/investigação individual de violência doméstica, sexual e/ou outras violências do Sinan. Os dados foram tabulados no Microsoft Excel 2010 e analisados por meio da estatística descritiva com medidas de frequência e percentuais. Segundo a Comissão Nacional de Ética em Pesquisa (CONEP) e Conselho Nacional de Saúde (CNS) pesquisas que envolvem apenas dados de domínio público que não permitam a identificação dos participantes não necessitam aprovação por parte do Sistema CEP-CONEP (http://conselho.saude.gov.br/).

\section{RESULTADOS E DISCUSSÃO}

O total de notificações em 2014 foi de 793. A notificação é uma das estratégias do Ministério da Saúde destinadas às ações de combate a violência e meio para articulação de políticas de saúde, sendo estabelecida como obrigatória, através da notificação compulsória dos casos de violência (VELOSO et al., 2013).

Entre as notificações, 40,48\% (321) foram do sexo masculino e 59,52 (472) feminino. Estudo realizado em Aracajú (SE) verificou que do total de vítimas da violência, $34,80 \%$ eram do sexo masculino contra $65,20 \%$ do sexo feminino (AGUIAR et al., 2015). Percentuais ainda mais distintos foram verificados em 
Portugal onde $76,10 \%$ dos casos, de violência contra a pessoa idosa, foram contra a mulher (GIL et al., 2015).

É possível refletir sobre a maior frequência de violência contra as mulheres sob o enfoque feminista. Compreender as dinâmicas de gênero significa buscar caminhos para a desconstrução dessas desigualdades no âmbito da saúde (CARDOSO, et al., 2015).

A maioria dos idosos foi da cor/raça branca com 73,27\% (581) seguido de cor/raça parda com 12,11 (96) (Figura 1), esses resultados vão de encontro ao observado na população geral do estado. De acordo com o IBGE $70,32 \%$ da população do Paraná é constituída da cor/raça branca (IBGE, 2010).

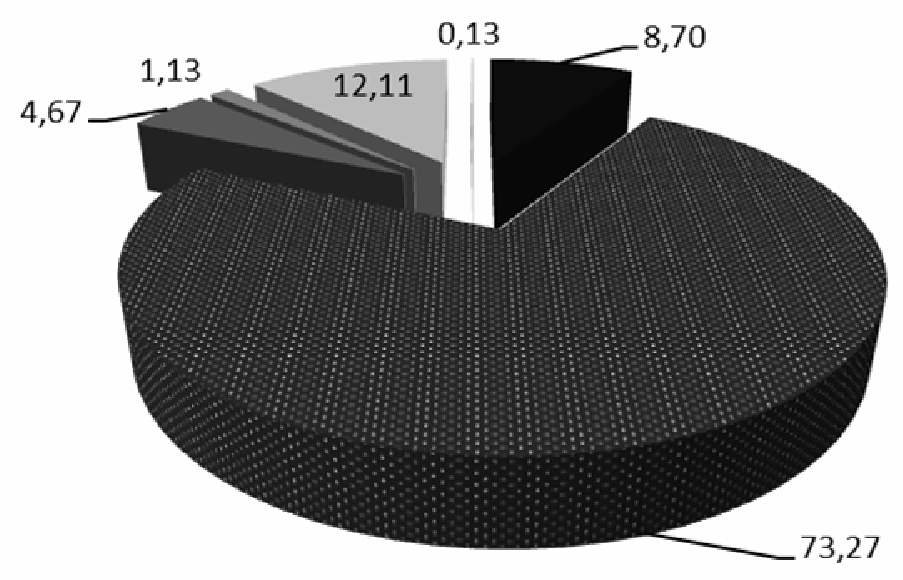

Ign/Branco Branca Preta $\square$ Amarela $\square$ Parda $\square$ Indigena

FIGURA 1. Distribuição das notificações de violência contra o idoso no Paraná segundo cor/raça, 2014.

Fonte: Sinan, 2017.

Em relação à escolaridade foi possível observar que 25,35\% (201) possuíam ensino fundamental incompleto, 3,40\% (27) ensino médio completo e 1,89\% (15) ensino superior completo. Aguiar et al. (2015) em estudo com idosos verificaram que mais da metade dos idosos vítima de violência $(66,1 \%)$ cursou o ensino fundamental e apenas $4,4 \%$ cursaram o ensino superior.

Santos-Orlandi et al. (2017) em estudo com idosos cuidadores referem que o período em que os idosos de hoje nasceram e cresceram, pode explicar os baixos níveis de escolaridade. Antigamente, início do século $X X$, a educação formal não era valorizada como hoje e as condições socioeconômicas precárias dificultavam 0 acesso às escolas, além de não haver incentivo por parte dos progenitores.

Do total de notificações, 50,57\% (400) dos casos se caracterizam por violência recorrente, ou seja, o idoso já sofreu violência anteriormente. Ao analisar o componente por sexo separado, verifica-se que entre os homens o percentual de violência recorrente foi de 29,25\% (117) e nas mulheres $70,75 \%$ (283). Este dado é importante, pois reflete como está sendo efetivo ou não, os programas de apoio a violência ao idoso (SHIMBO et al., 2011).

O local mais frequente, de ocorrência da violência foi a própria residência com $83,31 \%$ (659), seguido da via pública com 6,95\%(55). Outros estudos também 
apontam o domicílio como o espaço onde a violência predomina (AGUIAR et al., 2015; SOARES et al., 2015).

$\mathrm{Na}$ atenção básica, a equipe de saúde da família tem papel importante no reconhecimento da violência intrafamiliar contra o idoso, uma vez que permite aproximação com questões que envolvem a situação de violência (SHIMBO et al., 2011).

As esquipes da saúde da família orientam a participação comunitária e o controle social, criam condições e estimulam a participação dos usuários nos processos educativos, bem como nas avaliações e no planejamento da assistência ao idoso (SHIMBO et al., 2011). O reconhecimento da violência intrafamiliar pode auxiliar na minimização dos danos gerados em cada família e interromper a continuidade desse ciclo (SALIBA et al., 2007).

A Figura 2 mostra que o tipo de violência mais frequente foi a física com $37,68 \%$ (474), seguido de violência psicológica/moral com 26,55\% (334). Achados em um estudo de base populacional evidenciaram que os tipos de violência mais reportados foram física e a psicológica afetando $87,8 \%$ e $69,6 \%$ das pessoas estudadas, seguindo-se a violência financeira (47,5\%), sexual $(7,5 \%)$ e a negligência $(6,5 \%)$ (GIL et al., 2015). Estudo conduzido por Soares et al. (2015) em Aracajú encontrou dados que mostram negligência/abandono como principal tipo de violência, realidade distinta da verificada neste estudo.

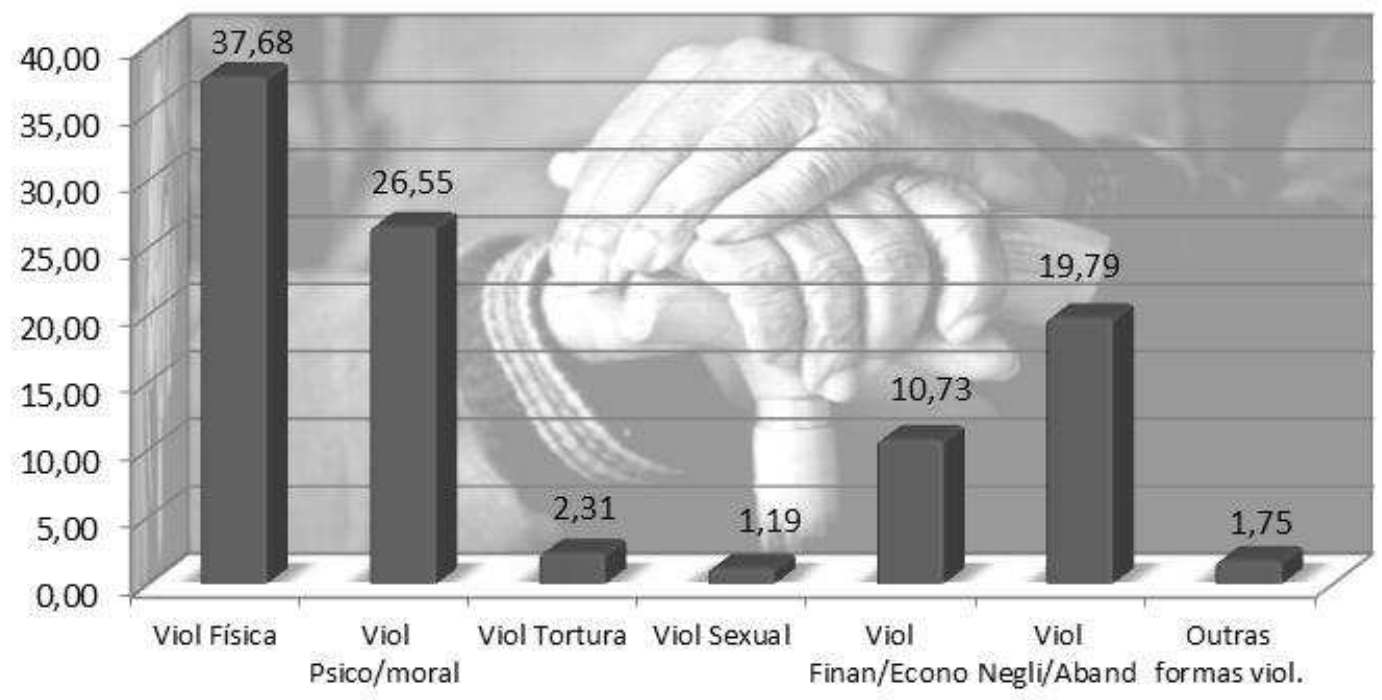

FIGURA 2. Distribuição das notificações de violência contra o idoso no Paraná, segundo tipos de violência, 2014.

Fonte: Sinan, 2017.

Quando se realiza a análise segundo sexo verifica-se que a mulher é sempre mais vulnerável do que o homem, independente do tipo de violência praticada, os percentuais de ocorrência dos agravos nas mulheres são muito mais elevados comparados ao homem (FIGURA 3). Revisão de literatura realizada sobre a temática verificou que muitos defendem uma maior vulnerabilidade da mulher idosa à violência, particularmente com aquelas que já sofriam violência doméstica em idade adulta, fato que exerce influência na ocorrência de maus-tratos (OLIVEIRA et al, 2013). 


\begin{tabular}{lrrrr} 
Tipos de violência & \multicolumn{2}{c}{ Masculino } & \multicolumn{2}{c}{ Feminino } \\
\cline { 2 - 5 } & \multicolumn{1}{c}{ N } & \multicolumn{1}{c}{$\%$} & \multicolumn{1}{c}{ N } & \multicolumn{1}{c}{$\%$} \\
\cline { 2 - 5 } Viol Física & 224 & 47,26 & 250 & 52,74 \\
Viol Psico/moral & 90 & 26,95 & 244 & 73,05 \\
Viol Tortura & 9 & 31,03 & 20 & 68,97 \\
Viol Sexual & 1 & 6,67 & 14 & 93,33 \\
Viol Finan/Econo & 35 & 25,93 & 100 & 74,07 \\
Viol Negli/Aband & 87 & 34,94 & 162 & 65,06 \\
Outras formas viol. & 9 & 40,91 & 13 & 59,09 \\
\hline
\end{tabular}

FIGURA 3. Distribuição das notificações de violência contra o idoso, segundo sexo. Paraná, 2014. Fonte: Sinan, 2017.

Dentre as formas de agressão na violência física, verifica-se que a mais comum é o uso de força corporal/espancamento com 50,30\% (334) dos casos notificados, seguido de ameaça com $24,40 \%$ (162). Estudo que analisou a violência doméstica contra o idoso no Brasil verificou que a principal forma de violência é o espancamento com $19,40 \%$ das notificações nos homens e $22,24 \%$ nas mulheres (MIZIARA et al., 2015). Em Portugal, as maiorias dos idosos violentados relatam terem sofrido mais de um tipo de violência (GIL et al., 2015).

Estudo realizado em uma delegacia especializada em Teresina, no Piauí, verificou que de acordo com as informações registradas nos boletins de ocorrência, agressão verbal e as ameaças são as mais frequentes entre os idosos, e os relatos destacam sentimento de medo, por parte do idoso (DAMASCENO et al., 2016).

É importante evidenciar sobre o ciclo da violência. Geralmente a violência segue um padrão de agressão. Os idosos atacados nem sempre são vítimas de agressão constante, nem a violência acontece fortuitamente. A agressão é inflingida em um ciclo repetitivo, composto de três frases: a criação da tensão, 0 ato de violência e uma fase amorosa, tranquila (PARAÍBA; SILVA, 2015).

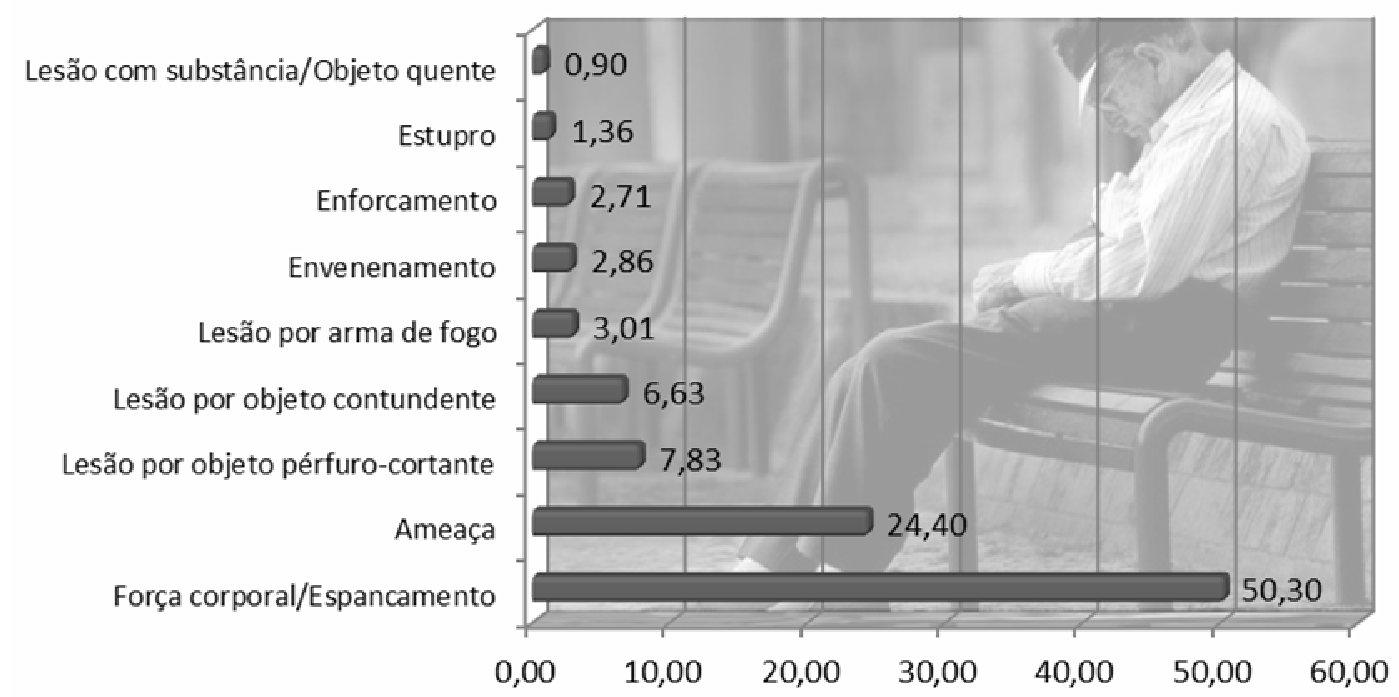

FIGURA 4. Distribuição das notificações de violência contra o idoso, segundo meio de agressão. Paraná, 2014.

Fonte: Sinan, 2017. 
A Figura 5 mostra que 46,18\% (314) dos idosos foram vítimas de seu filho(a). Outros estudos sobre o tema também verificaram que os agressores mais frequentes são os filhos (AGUIAR et al., 2015; SOARES et al. 2015). Em um contexto geral os agressores são pessoas conhecidas do idoso e de convívio mais próximo. Alves (2013) discute a complexidade que envolve o âmbito familiar, uma vez que este, cada vez mais, vem apresentando situações de conflitos e agressões relativos às questões de poder, autoridade e do papel que o idoso passa a ter nesse contexto.

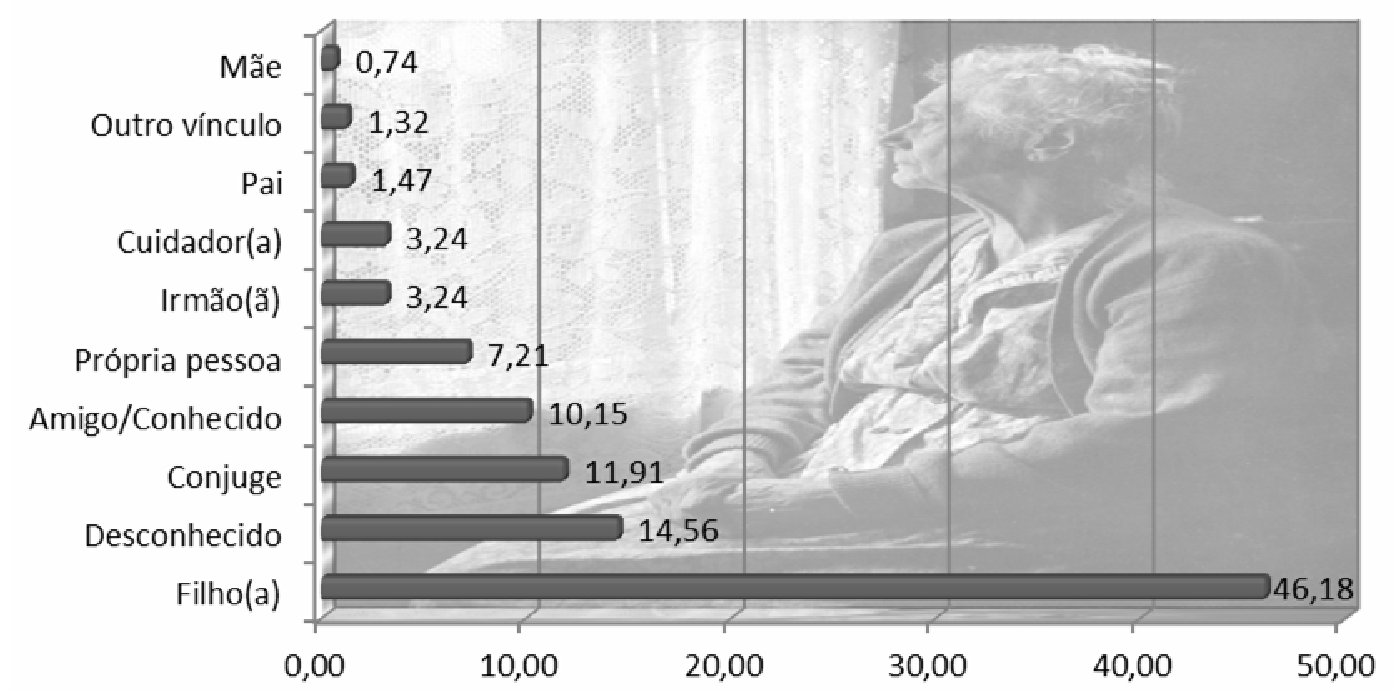

FIGURA 5. Distribuição das notificações de violência contra o idoso Fonte: Sinan, 2017. segundo agressor, Paraná 2014.

Após a ocorrência da violência, o encaminhamento dado foi ambulatorial em $36,95 \%$ (293) dos casos e hospitalar em 19,42\% (154). O desfecho foi alto em $76,42 \%$ (606) dos casos, óbito em decorrência da violência em 2,65\% (21) e evasão ou fuga em $0,63 \%$ (5). Embora a mulher seja mais vulnerável, verificou-se que as mortes são mais frequentes nos homens com 76,19\% (16) do total de óbitos. Este resultado é corroborado por estudo realizado no Brasil, que teve o intuito de evidenciar a violência domestica contra o idoso, onde $70,8 \%$ dos óbitos ocorreram no sexo masculino (APRATTO JÚNIOR, 2010).

Nesse sentido, um dos maiores desafios da saúde pública é promover saúde, qualidade de vida dos longevos (YAMAGUCHI et al., 2016). A Política Nacional de Promoção da Saúde (PNPS) como um dos temas prioritários a promoção da cultura da paz e de direitos humanos que busca promover, articular e mobilizar ações que estimulam a solidariedade, a convivência, o respeito à vida e o fortalecimento de vínculos, para desenvolver tecnologias sociais que auxiliam na mediação de conflitos diante de situações tensionais, a fim de reduzir as violências e construindo práticas solidárias e da cultura de paz (BRASIL, 2014).

\section{CONCLUSÃO}

Conclui-se que a violência contra o idoso no Paraná é bastante frequente, com uma elevada tendência em mulheres brancas de baixa escolaridade. Verificouse que o local de ocorrência mais comum foi o ambiente domiciliar tendo o filho(a) 
como principal agressor. A violência física foi o tipo mais observado através de força física/espancamento. O encaminhamento dos casos em sua maioria foi ambulatorial com evolução para alta, embora óbitos tenham ocorrido numa parcela menor.

A violência contra o idoso é um problema de saúde pública muitas vezes oculto na sociedade. Estudos desse tipo trazem a luz características importantes para compreensão do problema e podem auxiliar na elaboração e planejamento de ações e políticas públicas destinadas ao controle e redução desse problema social e de saúde pública. Ações de promoção de saúde e proteção desse grupo podem ser aprimoradas e veiculadas de modo a alterar a realidade presente. O impacto desse problema transcende 0 aspecto físico, se estende às relações familiares e de amigos no contexto principalmente domiciliar. A pessoa idosa é vulnerável e carece de medidas para sua proteção. Outros estudos que forneçam dados de violência em outras localidades podem auxiliar na identificação de padrões de ocorrência e formas de intervir. É possível que exista uma subnotificação importante visto que as dinâmica e relações familiares são complexas. Estudos nessa temática continuam relevantes, pois se trata de um problema frequente e que pode vir a apresentar um aumento vertiginoso em decorrência do aumento da população idosa. Frente a isso, conhecer aspectos relativos a esse agravo instrumentaliza os profissionais a lidarem com o problema e buscarem ações para reduzi-lo.

\section{AGRADECIMENTOS}

Ao Centro Universitário de Maringá (UNICESUMAR), Instituto Cesumar de Ciência, Tecnologia e Inovação (ICETI) e à Coordenação de Aperfeiçoamento de Pessoal de Nível Superior (CAPES) que contribuíram com este estudo.

\section{REFERÊNCIAS}

ACIERNO, R.; HERNANDEZ, M. A.; AMSTADTER, A. B.; RESNICK, H. S.; STEVE, K. et al. Prevalence and Correlates of Emotional, Physical, Sexual, and Financial Abuse and Potential Neglect in the United States: The National Elder Mistrat-ment Study. American Public Health Association, v. 100, n, 2, p. 292-297, 2010. DOI: http://dx.doi.org/10.2105/AJPH.2009.163089.

AGUIAR, M. P. C.; LEITE, H. A.; DIAS, I. M.; MATTOS, M. C. T., LIMA, W. R. Violência contra idosos: descrição de casos no Município de Aracaju, Sergipe. Escola Anna Nery, Rio de Janeiro, v. 19, p. 343-349, 2015. Disponível em:http://www.redalyc.org/articulo.oa?id=127739655022 DOI: $10.5935 / 1414$ 8145.20150047

ALVES, C. Relações familiares e violência: idosos entre abafos e desabafos. Fazendo Gênero, v. 8, p.1-6, 2013. Disponível em: http://fazendogenero.ufsc.br/8/sts/ST68/Carla_Maria_Lobato_Alves_68.pdf

APRATTO JÚNIOR, P. C. A violência doméstica contra idosos nas áreas de abrangência do Programa Saúde da Família de Niterói (RJ, Brasil). Ciência \& Saúde Coletiva, v. 15, n. 6, p. 2983-2995, 2010. DOI: http://dx.doi.org/10.1590/S1413-81232010000600037 
BRASIL. Ministério da Saúde (MS). Portaria n 2.446 MS/GM, de 11 de novembro de 2014. Redefine a Política Nacional de Promoção da Saúde. Diário Oficial da União 2014; 12 nov. Disponível em: http://bvsms.saude.gov.br/bvs/saudelegis/gm/2014/prt2446_11_11_2014.html

CACHINA, A. M. P.; PAIVA, L. L.; TORRES, T. L. Violência intrafamiliar contra idosos: Revisão sistemática. Liberabit, v. 22, n. 2, p. 185-196, 2016. Disponível em: http://www.scielo.org.pe/pdf/liber/v22n2/a06v22n2.pdf

CARDOSO DUARTE, M.; GODOY SERPA DA FONSECA, R. M.; DE SOUZA, V. DUMONT PENA, E. Gênero e violência contra a mulher na literatura de enfermagem: uma revisão. Revista Brasileira de Enfermagem, v. 68, n. 2, p. 325332, 2015. Disponível em: http://www.redalyc.org/articulo.oa?id=267040408020. DOI: http://dx.doi.org/10.1590/0034-7167.2015680220i.

DAMASCENO, C. K. C. S.; SOUZA, C. M. M.; MOURA, M. E. B. Violência contra pessoas idosas registrada em delegacia especializada de segurança e proteção ao idoso. Rev enferm UFPE on line, Recife, v. 10, n. 3, p. 949-57, 2016. Disponível em: https://periodicos.ufpe.br/revistas/revistaenfermagem/article/view/11045/12451. DOI: 10.5205/reuol.8702-76273-4-SM.1003201602

DUQUE, A. M.; LEAL, M. C. C.; MARQUES, A. P. O.; ESKINAZI, F. M. V.; DUQUE, A; M. Violência contra idosos no ambiente doméstico: prevalência e fatores associados (Recife/PE). Ciência \& Saúde Coletiva, v. 17, n. 8, p. 2199-2208, 2012. DOI: http://dx.doi.org/10.1590/S1413-81232012000800030

GIL, A. P.; SANTOS, A. J.; KISLAYA I.; SANTOS, C.; MASCOLI L. et al. Estudio sobre las víctimas de la violencia en Portugal: sociografía de la ocurrencia. Caderno de Saúde Pública, Rio de Janeiro, v. 31, n. 6, p. 1234-1246, 2015. Disponível em: http://www.scielosp.org/scielo.php?script=sci_arttext\&pid=S0102-

311X2015000601234\&lng=en. DOI: http://dx.doi.org/10.1590/0102-311X00084614.

IBGE. CENSO 2010. Disponível em:< http://www. censo2010. ibge. gov. br/. 2010.

IMSPM. Instituto de Mayores y Servicios Sociales Malos tratos a personas mayores. Organização Mundial de Saúde: Madrid; Espanha. 2007.Disponível em: http://www.who.int/mediacentre/factsheets/fs357/es/

MASCARENHAS, M. D. M.; ANDRADE, S. S. C. A.; NEVES, A. C. M. N.; PEDROSA, A. A. G.; SILVA, M. M. A. et al. Violence against the elderly: analysis of the reports made in the health sector - Brazil. Ciência \& Saúde Coletiva, v. 17, n. 9, p. 2331-2341, 2012. DOI: http://dx.doi.org/10.1590/S1413-81232012000900014

MIZIARA, C. S. M. G.; BRAGA, M. V.; CARVALHO, F. I.; TEIXEIRA, T. V.; MIZIARA, I. D. et al. Vítima silenciosa: violência doméstica contra o idoso no Brasil. Saúde, Ética \& Justiça. São Paulo, v. 20, n. 1, p. 1-8, 2015. Disponível em: http://www.journals.usp.br/sej/article/view/102816.

DOI: http://dx.doi.org/10.11606/issn.2317-2770.v20i1 
OLIVEIRA, A. A. V.; TRIGUEIRO, D. R. S. G.; FERNANDES, M. G. M., SILVA, A. O. Maus-tratos a idosos: revisão integrativa da literatura. Revista Brasileira de Enfermagem, Brasília, v. 66, n. 1, p.128-133, 2013. Disponível em: $<$ http://www.scielo.br/scielo.php?script=sci_arttext\&pid=S0034-

71672013000100020\&lng=en\&nrm=iso >. $\quad$ DOI: http://dx.doi.org/10.1590/S003471672013000100020.

PAIVA, M. M.; TAVARES, D. M. S. Physical and psychological violence against the elderly: prevalence and associated factors. Revista Brasileira de Enfermagem, v. 68, n. 6, p. 1035-1041, 2015. DOI: http://dx.doi.org/10.1590/0034-7167.2015680606i

PARAÍBA, P. M. F.; SILVA, M. C. M. Perfil da violência contra a pessoa idosa na cidade do Recife-PE. Revista Brasileira de Geriatria e Gerontologia, v. 18, n. 2, p. 295-306, 2015. DOI: http://dx.doi.org/10.1590/1809-9823.2015.14047

SALIBA, O.; GARBIN, C. A. S.; GARBIN, A. J. I.; DOSSI, A. P. Responsabilidade do profissional de saúde sobre a notificação de casos de violência doméstica. Revista de Saúde Pública, v. 41, n. 3, p. 472-477, 2007. DOI: http://dx.doi.org/10.1590/S0034-89102007000300021.

SAMPAIO, L. S.; FERREIRA, M. J. S., SAMPAIO, T. S. O., SOUZA, W. P.; PRADO, A. P. S. et al. Violência física em idosos. C\&D-Revista Eletrônica da FAINOR, Vitória da Conquista, v.10, n.2 p.188-200, 2017. Disponível em: http://srv02.fainor.com.br/revista/index.php/memorias/article/view/661/337

SANTOS-ORLANDI, A. A.; PEREIRA DE BRITO, T. R.; OTTAVIANI, A. C.; ROSSETTI, E. S.; ZAZZETTA, M. S. et al. Perfil de idosos que cuidam de outros idosos em contexto de alta vulnerabilidade social. Escola Anna Nery, Rio de Janeiro, $\quad$ v. 21, p. 1-8, 2017. Disponível em: <http://www.redalyc.org/articulo.oa?id=127749356013> DOI: http://dx.doi.org/10.5935/1414-8145.20170013.

SHIMBO, A. Y.; LABRONICI, L. M.; MANTOVANI, M. F. Reconhecimento da violência intrafamiliar contra idosos pela equipe da estratégia saúde da família. Escola Anna Nery Revista de Enfermagem, v. 15, n. 3, p. 506-510, 2011. Disponível em: http://www.scielo.br/pdf/ean/v15n3/a09v15n3

SIEGEL, M.; MAZHEIKA, Y.; MENNICKEN, R.; RITZ-TIMME, S.; GRAß, H. et al. Because we feel, we have to do something"-Barriers in the prevention of violence and key areas requiring action: a qualitative interview study with professional nurses and managers in the field of elderly care. Zeitschrift fur Gerontologie, v. 21, p. 1-6, 2017. DOI: https://doi.org/10.1007/s00391-017-1228-0

SOARES, A. C. G. M.; SANTOS, E. M.; FONSECA, V.; OLIVEIRA, L. G. F. Perfil epidemiológico da violência contra o idoso no município de Aracaju. Interfaces Científicas - Humanas e Sociais, Aracaju, v. 3, n. 2, p. 109-120, 2015. Disponível em: $\quad$ https://periodicos.set.edu.br/index.php/humanas/article/view/1664. DOI: http://dx.doi.org/10.17564/2316-3801.2015v3n2p109-120 
SOUSA, R. I. M.; BERNARDINO, Í. M.; CASTRO, R. D.; CAVALCANTI, A. L.; BENTO, P. M.; et al. Facial trauma as physical violence markers against elderly Brazilians: A comparative analysis between genders. Archives of Gerontology and Geriatrics, v. 67, p. 55-60, 2016. DOI: https://doi.org/10.1016/j.archger.2016.06.015

VELOSO, M. M. X.; MAGALHÃES, C. M. C. ; DELL'AGLIO, D. D. A.; CABRAL, I. R.; GOMES, M. M. Notificação da violência como estratégia de vigilância em saúde: perfil de uma metrópole do Brasil. Ciência \& Saúde Coletiva, v. 18, n. 5, p. 12631272, $2013 . \quad$ Disponível em: HTTP://WWW.SCIELO.BR/SCIELO.PHP?SCRIPT=SCI_ARTTEXT\&PID=S141381232013000500011\&LNG=EN\&NRM=ISO. DOI: HTTP://DX.DOI.ORG/10.1590/S1413-81232013000500011.

WANDERBROOCKE, A. C. N. D. S.; MORÉ, C. L. O. O. Abordagem profissional da violência familiar contra o idoso em uma unidade básica de saúde. Cadernos de Saúde Pública, v. 29, n. 12, p. 2513-2522, 2013. DOI: http://dx.doi.org/10.1590/0102-311X00174112

WANDERBROOCKE, A. C. N. D. S.; MORÉ, C. L. O. O. Significados de violência familiar contra o idoso na perspectiva de profissionais da Atenção Primária à Saúde. Ciência \& Saúde Coletiva, v. 17, n. 8, p. 2095-2103, 2012. DOI: http://dx.doi.org/10.1590/S1413-81232012000800020

WARMLING, D.; LINDNER, S. R.; COELHO, E. B. S. Intimate partner violence prevalence in the elderly and associated factors: systematic review. Ciência \& Saúde Coletiva, v. 22, n. 9, p. 3111-3125, 2017. DOI: http://dx.doi.org/10.1590/1413-81232017229.12312017

YAMAGUCHI, M. U.; BERNUCI, M. P.; \& PAVANELLI, G. C. Scientific research about the National Policy for Health Promotion. Ciência \& Saúde Coletiva, v. 21, n. 1. 6, p. 1727-1736, 2016. DOI: http://dx.doi.org/10.1590/1413-81232015216.07462016 